\title{
Incorporation of dengue virus replicon into virus-like particles by a cell line stably expressing precursor membrane and envelope proteins of dengue virus type 2
}

\author{
Chih-Yun Lai ${ }^{1}$, Hsien-Ping $\mathrm{Hu}^{1}$, Chwan-Chuen King $^{2}$ \& Wei-Kung Wang ${ }^{1,3, *}$ \\ ${ }^{1}$ Institute of Microbiology, College of Medicine, National Taiwan University, No.1 Sec.1 Jen-Ai Rd, Taipei, \\ Taiwan; ${ }^{2}$ Institute of Epidemiology, College of Public Health, National Taiwan University, Taipei, Taiwan; \\ ${ }^{3}$ Department of Internal Medicine, College of Medicine, National Taiwan University, Taipei, Taiwan
}

Received 10 May 2007; accepted 8 August 2007

(c) 2007 National Science Council, Taipei

Key words: dengue virus (DENV), replicon, virus-like particles (VLPs)

\begin{abstract}
While virus-like particles (VLPs) containing subgenomic replicons, which can transduce replicons into target cells efficiently for studying viral replication and vectors of gene therapy and vaccine, have been established for several flaviviruses, none has been reported for the four serotypes of dengue virus, the causal agent of the most important arboviral diseases in this century. In this study, we successfully established a cell line stably expressing the precursor membrane/envelope (PrM/E) proteins of dengue virus type 2 (DENV2), which can package a DENV2 replicon with deletion of $\operatorname{PrM} / \mathrm{E}$ genes and produce single-round infectious VLPs. Moreover, it can package a similar replicon of different serotype, dengue virus type 4, and produce infectious chimeric VLPs. To our knowledge, this study reports for the first time replicon-containing VLPs of dengue virus. Moreover, this convenient system has potential as a valuable tool to study encapsidation of dengue virus and to develop novel chimeric VLPs containing dengue virus replicon as vaccine in the future.
\end{abstract}

\section{Introduction}

Dengue viruses belong to the genus Flavivirus in the family Flaviviridae. Among the 70 or so arthropod-borne flaviviruses, the four serotypes of dengue viruses (DENV1, DENV2, DENV3, and DENV4) cause the most important arboviral disease in the tropical and subtropical areas [1, 2]. It has been estimated that more than 2.5 billion people in over 100 countries are at risk of infection, and approximately 100 million dengue infections occur annually throughout the world [1-3]. After dengue virus infection, an individual may be asymptomatic, present a relatively mild disease, dengue fever, or develop severe and potentially life-threatening diseases, dengue

*To whom correspondence should be addressed. Fax: + 886-223915293; E-mail: wwang60@yahoo.com hemorrhagic fever/dengue shock syndrome. Without early diagnosis and appropriate treatment, the mortality of DHF/DSS could be as high as $10-15 \%$ [1-3].

Dengue virus contains a positive-sense, singlestranded RNA genome of approximately 10,600 bases in length. Flanked by the $5^{\prime}$ and $3^{\prime}$ untranslated regions (UTR), the single open reading frame of the genome encodes a polyprotein precursor, which is subsequently cleaved by cellular and viral protease into three structural proteins, the capsid (C), precursor membrane (PrM), and envelope (E), as well as seven non-structural (NS) proteins, NS1, NS2A, NS2B, NS3, NS4A, NS4B and NS5 [4]. A common feature in the replication of flaviviruses is the assembly of structural proteins, PrM and E, into subviral particles [4]. Co-expression of PrM and $\mathrm{E}$ proteins in the absence of $\mathrm{C}$ protein can generate recombinant subviral particles 
(RSPs), which have been shown to be similar to the infectious virions in the structural and functional organization [5-7].

Subgenomic replicons of positive-stranded RNA viruses, which contain all the non-structural proteins required for amplifying themselves but lack some or all of the structural proteins, have been shown to be useful tools to study viral replication in the absence of virion assembly and maturation [8-11]. Because of the unique features including high level of expression, no DNA intermediate, exclusively cytoplasmic replication, and relative ease of construction, subgenomic replicons offered great potential as vectors for gene expression and vaccine development $[9,12$, 13]. Subgenomic replicons of several members of flaviviruses including Kunjin virus, West Nile virus, tick-borne encephalitis virus, yellow fever virus and dengue virus were established [14-20]. Most of these subgenomic replicons have been recently reported to incorporate into virus-like particles (VLPs) as a means of efficient transduction into target cells with the exception of dengue virus [14, 17, 21-27]. In this study, we established a cell line stably expressing the $\operatorname{PrM} / E$ proteins of DENV2, which can successfully encapsidate the DENV2 replicon and produce single-round infectious VLPs. Moreover, this cell line can also encapsidate the DENV4 replicon and produce infectious chimeric VLPs, which contain the C protein and replicon RNA of DENV4 and the $\mathrm{PrM} / \mathrm{E}$ proteins of DENV2.

\section{Materials and methods}

\section{Generation of replicon constructs}

To construct the DENV2 replicon $\mathrm{p} 2 \mathrm{C} 102$, a PCR fragment was amplified by using plasmid $\mathrm{p} 2$, a fulllength cDNA clone of DENV2 (Tonga/74 strain) (kindly provided by Dr. S. S. Whitehead), as template and the primer pairs, p2AscIASP6 (5'-TTTCCGGGCGCGCCGATTTAGG-3') and p2C102BSpeI (5'-CTAG ACTAGTG CTACGTGA TACAGTTCTACGTCTCCTGTTTAAG-3'), cut with $A s c \mathrm{I}$ and $S p e \mathrm{I}$, and cloned into respective sites of $\mathrm{p} 2$ [28].

To construct the DENV4 replicon $\mathrm{p} 4 \mathrm{C} 102$, a PCR fragment was amplified by using p2AXhoI, a full-length cDNA clone of DENV4 (814,669 strain) (kindly provided by Dr. C. J. Lai), as template and the primer pairs, d4C1ABglII (5'-GAGCAGATCTCTGGAAAAATG-3') and d4C102BXhol (5'-CAGGGAGCT CAACGTTGA CCTTTTTCTCC-3'), cut with BglII and XhoI, and cloned into $\mathrm{T} 7 \mathrm{p}^{\prime} 5-2$, which was modified from $\mathrm{p}^{\prime} 5-2$ (5' cDNA clone of p2AXhoI, also provided by Dr. C. J. Lai) by replacing the SP6 promoter with $\mathrm{T} 7$ promoter $[29,30]$. The resulting plasmid T7C102p'5-2 was cut with BamHI and XhoI and cloned into pFastBacl vector (Invitrogen, Carlsbad, CA) to generate T7C102pFast. The $3^{\prime}$ cDNA clone of p2AXhoI was cut out with XhoI and $K p n \mathrm{I}$, and cloned into the respective sites of T7C102pFast to obtain $\mathrm{p} 4 \mathrm{C} 102$. To construct the DENV4 replicon p4C30, a PCR fragment was amplified by using p2AXhoI as template and the primer pairs, d4C1ABglII and d4C30BXhol (5'AAACCTCGAGTTTGTGGAT CTCTTCCGCC CCCCTTGAGGGGTTGA-3'), cut with BglII and XhoI, and cloned into T7p'5-2 by replacing the SP6 promoter with T7 promoter [29, 30]. The resulting plasmid T7C30p'5-2 was cut with BamHI and $X h o I$ and cloned into pFastBacl vector (Invitrogen, Carlsbad, CA) to generate T7C30pFast. The $3^{\prime}$ cDNA clone of p2AXhoI was cut out with $X h o \mathrm{I}$ and $K p n I$, and cloned into the respective sites of $\mathrm{T} 7 \mathrm{C} 30 \mathrm{pFast}$ to obtain p4C30. All the constructs have been confirmed by sequencing at the junctions with the BigDye terminator cycle sequencing kit and the ABI 3730 automated sequencer (Applied Biosystems, Foster City, CA).

\section{In vitro transcription and RNA transfection}

Replicon plasmid was linealized with $K p n \mathrm{I}$, ethanol precipitated, and in vitro transcribed by using the Epicentre AmpliCap T7 and SP6 high yield message marker kits (Epicentre Biotechnologies, Madison, WI). RNA was further purified using the Qiagen RNeasy mini kit (Qiagen, Valencia, CA), followed by quantification with a spectrophotometer, and then introduced into cells by electroporation. Approximately $3.2 \times 10^{6}$ cells in $800 \mu \mathrm{l}$ serum free DMEM medium was electroporated with $30-50 \mu \mathrm{g}$ of in vitro transcribed RNA in $4 \mathrm{~mm}$ cuvettes using the BTX electro cell manipulator $^{\text {TM }}$ (BTX, Holliston, MA) with one pulse at the setting of $270 \mathrm{~V}, 800 \mu \mathrm{F}$, and $75 \Omega$. After being 
chilled on ice for $10 \mathrm{~min}$, the cells were transferred to $10 \mathrm{~cm}$ culture dishes.

\section{Detection of negative sense dengue viral RNA}

Total RNA from transfected cells was extracted with the Qiagen RNeasy mini kit (Qiagen, Valencia, CA) and an aliquot of RNA was subjected to no RT or RT by using SuperScript II reverse transcriptase (Invitrogen, Carlsbad, CA) and sense primers specific to the $3^{\prime} \mathrm{UTR}$ region of DENV2 (5'-CTGTAGTCTCACTGGAAGGAC-3') (genome positions 10553-10573, Tonga/74 strain) [28] or of DENV4 (5'-CTGTACGCGTGGCATATT GGAC-3') (genome positions 10398-10419, 814669 strain) [29]. PCR was then performed with primer pairs covering the regions in $3^{\prime} \mathrm{UTR}$ to generate a 110 -bp product (for DENV2) or a 200 bp product (for DENV4). The sequences of the primer pairs were: 5'-TTAGAGGAGACCCCCC C-3' (positions 10580-10596) and 5'-CATTCCAT TTTCTGGCGTCCT-3' (positions 10690-10670) for DENV2; 5'-TTAGAGGAGACCCCTCCC-3' (positions 10426-10443) and 5'-CAATCCATCTT GCGGCGCTCT-3' (positions 10626-10606) for DENV4. Besides, an aliquot of extracted total RNA was subjected to the Superscript II one-step RT-PCR system (Invitrogen, San Diego, CA) by using the primers, 5'-GTGATGGTGGGCATGG GTCA-3' and 5'-ACGTACATG GCTGGGGTG TT- $3^{\prime}$, to generate a 275 -bp product of $\beta$-actin as internal control.

\section{Western blot analysis}

After transfection of the replicon RNA, cells were harvested, washed twice with $1 \times$ phosphate-buffered saline (PBS), and lysed with $200 \mu \mathrm{l}$ freshly prepared lysis buffer (1\% NP-40, $50 \mathrm{mM}$ Tris $\mathrm{pH}$ $8.0,150 \mathrm{mM} \mathrm{NaCl}, 2 \mathrm{mM}$ EDTA, and $1 \mathrm{mM}$ $\mathrm{Na}_{3} \mathrm{VO}_{4}$ ). Samples were mixed with equal volumes of loading buffer $(2 \%$ SDS, $0.5 \mathrm{M}$ Tris $\mathrm{pH} 6.8$, $20 \%$ glycerol, $0.001 \%$ bromophenol blue), boiled at $95{ }^{\circ} \mathrm{C}$ for $2 \mathrm{~min}$, and subjected to $12 \%$ SDSpolyacryamide gel electrophoresis. After transfer and blocking, the nitrocellulose membrane (Hybond-c super, Amersham Biosciences, UK) was incubated with primary antibody including mouse monoclonal antibody (MAb) against NS1 protein (DB29-1, kindly provided by Dr. H. C. Wu), serum from dengue patients or rabbit polyclonal serum against $\mathrm{C}$ protein, followed by relevant horseradish peroxidase-conjugated secondary antibodies (Pierce, Rockford, IL). The signals were detected by enhanced chemiluminescence reagents (PerkinElmer life sciences, Boston, MA) [31].

\section{Indirect immunofluorescence assay (IFA)}

After DNA or RNA transfection or infection with VLPs, cells were spotted onto 10-well Tefloncoated slides, air dried, and fixed with cold acetone at $-20{ }^{\circ} \mathrm{C}$ for $20 \mathrm{~min}$. The slides were incubated at $37^{\circ} \mathrm{C}$ for $1 \mathrm{~h}$ with the first antibody (DB29-1 or 4G2) (American Type Culture Collection, Rockville, MD) [32], washed with $1 \times$ PBS three times, followed by incubation with the secondary antibody, fluorescein isothiocyanate (FITC)-conjugated goat anti-mouse IgG (Kirkegaard \& Perry Laboratories, Gaithersburg, MD). Following final washing, drying, and mounting, the slides were observed under a fluorescence microscope.

\section{Establishment of a stable clone expressing $\operatorname{Pr} M / E$} proteins of DENV2

Plasmid pCDJssD2J396 was modified from plasmid pCB8D2-2J-2-9-1 (kindly provided by Dr. G. J. J. Chang) [33], which expressed the signal peptide of Japanese encephalitis virus (JEV), PrM protein of DENV2, and chimeric E protein containing the $\mathrm{N}$-terminal 395 residues of DENV2 and C-terminal 100 residues of JEV (the stemanchor region of the $\mathrm{E}$ protein), by cloning the KpnI-NotI fragment into the respective sites of a JEV PrM/E expression plasmid with neomycin phosphotransferase gene, pCDJE2-7 (also provided by Dr. G. J. J. Chang) [34]. Plasmid pCB8D2-2J-2-9-1 was reported to produce RSPs efficiently [33]. 293 cells were grown in Dulbecco's modified eagle's medium (DMEM) supplemented with $10 \%$ fetal calf serum, $1 \%$ HEPES, $1 \%$ penicillin/streptomycin and $1 \%$ L-glutamine, and transfected with $2 \mu \mathrm{g}$ of $\mathrm{PrM} / \mathrm{E}$ expression plasmid of DENV2 that contained the neomycin phosphotransferase gene, pCDJssD2J396, by calcium phosphate method [35]. One day later, the cells were subjected to G418 selection $(800 \mu \mathrm{g} / \mu \mathrm{l})$ in DMEM with the medium and G418 replaced every 34 days. After 2-3 weeks, cells grown from foci were transferred to a T-25 flask and subsequently to a T-75 flask. Cells were passaged under G418 
selection for one more week and then stored in liquid nitrogen until use.

\section{Infectivity and characterization of VLPS}

At approximately day 10 post-transfection of replicon RNA to stable clones, culture supernatants containing VLPs collected from two $10 \mathrm{~cm}$ dishes were clarified by centrifugation at 2,500 rpm and $4{ }^{\circ} \mathrm{C}$ for $20 \mathrm{~min}$ and subjected to $20 \%$ sucrose cushion ultracentrifugation at 19,000 rpm (SW28 rotor, Beckman, Fullerton, CA) at $4{ }^{\circ} \mathrm{C}$ for $5 \mathrm{~h}$. The pellets containing VLPs were resuspended in $1 \mathrm{ml}$ RPMI medium and filtered through a $0.22-\mu \mathrm{m}$ pore-sized membrane, and an aliquot $(800 \mu \mathrm{l})$ were inoculated to BHK21 cells in 24-well plates. BHK-21 cells were grown in RPMI medium supplemented with 5\% fetal calf serum, $1 \%$ penicillin/streptomycin and $1 \%$ Lglutamine. After $48 \mathrm{~h}$, expression of NS1 protein in BHK-21 cells was examined by IFA (see above). The remaining $200 \mu \mathrm{l}$ was further subjected to centrifugation at $14,000 \mathrm{rpm}$ (851 rotor, Thermo electron corporation, Walthom, MA) at $4{ }^{\circ} \mathrm{C}$ for $1 \mathrm{~h}$ to obtain the pellet, which was resuspended in $20 \mu \mathrm{TNE}$ buffer $(50 \mathrm{mM}$ Tris- $\mathrm{HCl}, 1 \mathrm{M} \mathrm{NaCl}$, $10 \mathrm{mM}$ EDTA) and subjected to Western blot analysis and RNA extraction with Qiagen viral RNA kit (Qiagen, Valencia, CA). RT and PCR were preformed targeting two regions, the $3^{\prime} \mathrm{UTR}$ by using an antisense primer specific to $3^{\prime} \mathrm{UTR}$ of DENV4, 5'-CCTACCGGTACCAGAACC-3' (genome positions 10661-10644) [29] and PCR primers for DENV4 described above, and the C gene by using a DENV4-specific antisense primer, T7C102BXhoI, and PCR primers described previously [36]. An aliquot of the pellets was added to a 300 mesh formvar/carbon film, stained with saturated uranyl acetate (Electron Microscopy Sciences, Washington, PA), and examined by electron microscope.

\section{MTT cytotoxicity assay}

About $6.25 \times 10^{4}$ of 293 cells and cells from the established D2-PrM/E clone were prepared in 96well plates and assessed in triplicates by an MTT assay at various time after thawing $(\mathrm{R} \& \mathrm{D}$ systems, Minneapolis, MN). Briefly, cells in each well were incubated with $10 \mu \mathrm{l}$ of MTT reagent [3'(4,5-dimethylthiazol-yl)-2, 5'-diphenyl tetrazolium bromide] at $37{ }^{\circ} \mathrm{C}$ for $2.5 \mathrm{~h}$ until purple precipitate was clearly visible by light microscopy. After adding detergent, cells were further incubated at room temperature in the dark to ensure dissolution of all formazan crystals. Cell viability was the percentage of the absorbance at $570 \mathrm{~nm}$ of $\mathrm{D} 2-\operatorname{PrM} / \mathrm{E}$ clone relative to that of the parental 293 cells.

\section{Results}

Our strategy of generating dengue VLPs capable of encapsidating homologous and heterologous replicons included three steps. First, establishment of a stable cell line producing RSPs of DENV2. Second, construction of DENV2 subgenomic replicon and transfection into the stable cell line to generate DENV2 VLPs containing DENV2 replicon. Third, construction of DENV4 subgenomic replicon and transfection into the stable cell line to generate DENV2 VLPs containing DENV4 replicon.

\section{Establishment of a cell line constitutively producing RSPS of DENV2}

To generate a cell line stably expressing $\operatorname{PrM} / \mathrm{E}$ proteins and producing RSPs of DENV2, we constructed pCDJssD2J396, which contained the neomycin phosphotransferase gene and a chimeric $\operatorname{PrM} / E$ gene encoding the signal sequence of JEV, PrM protein and N-terminal 395 residues of $\mathrm{E}$ protein of DENV2 and C-terminal 100 residues of E protein of JEV (Figure 1A). This construct was modified from pCB8D2-2J-2-9-1, which was previously reported to produce RSPs of DENV2 efficiently [33]. After 2-3 weeks selection with G418, the pCDJssD2J396(DNA)-transfected 293 cells, now designated as D2-PrM/E clone, were subjected to IFA by using anti-dengue E MAb. As shown in Figure 1B, more than $90 \%$ positive cells were found in the D2-PrM/E clone but not in the mock-transfected cells. Western blot analysis of cell lysates derived from D2-PrM/E clone revealed readily detectable PrM and $\mathrm{E}$ proteins, indicating that expression of $\operatorname{PrM} / \mathrm{E}$ proteins was intact (Figure 1C, left). To examine the production of RSPs, culture supernatants derived from D2-PrM/ E clone was subjected to $20 \%$ sucrose cushion 

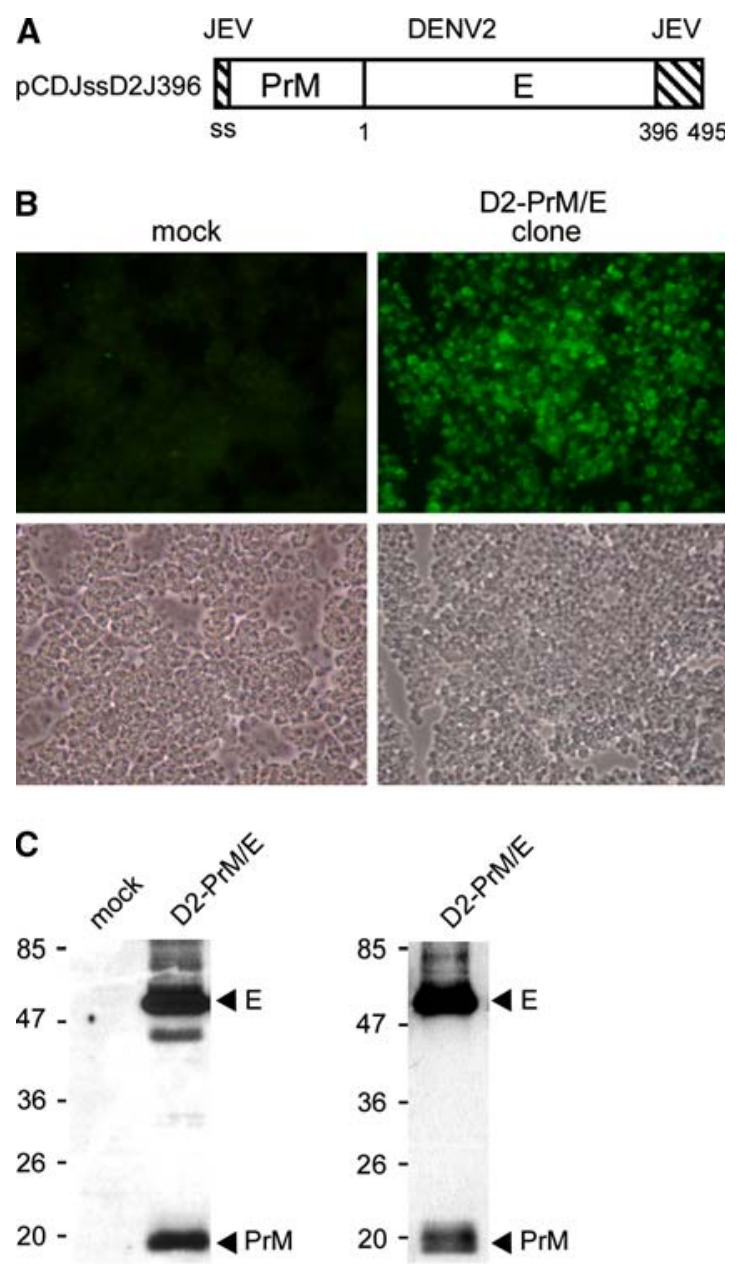

cell lysates

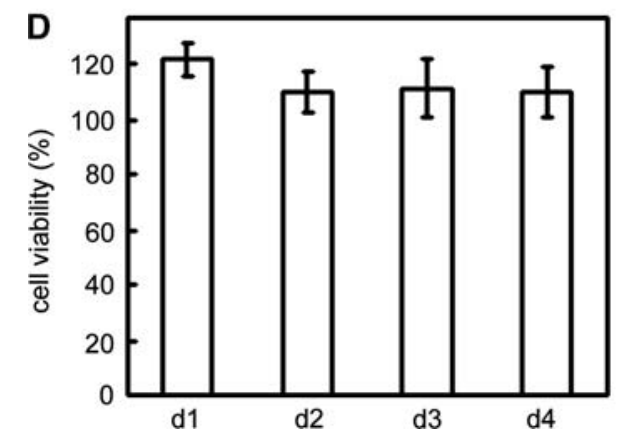

ultracentrifugation and the pellets to Western blot analysis. As shown in Figure 1C, large mounts of $\operatorname{PrM}$ and $\mathrm{E}$ proteins were detected, suggesting efficient production of RSPs by the D2-PrM/E clone. After 15 passages of this clone, there was
Figure 1. Establishment of a cell line stably expressing $\operatorname{PrM} / \mathrm{E}$ proteins of DENV2. (A) Schematic diagram of the construct, pCDJssD2J396, which encodes the PrM/E proteins of DENV2 with the signal sequence (ss) and C-terminal 100 residues (residues 396-495) of E protein replaced by JEV. (B) Expression of E protein by the DENV2 PrM/E stable clone. After 2-3 weeks of selection with G418, the pCDJssD2J396(DNA)-transfected 293 cells, designated as D2-PrM/E clone, were subjected to IFA using anti-E MAb, 4G2 [31]. (C) Expression of PrM/E proteins and production of RSPs by the D2-PrM/E stable clone. Cell lysates (left) and pellets lysates (right) derived from culture supernatants of the D2-PrM/E clone were subjected to Western blot analysis by using serum from dengue patient [30]. Arrow heads indicate PrM and E proteins. The size of molecular weight marker is shown in $\mathrm{kDa}$. (D) Cell viability of the established D2-PrM/E clone was assessed in triplicates by an MTT assay at various time after thawing of the D2-PrM/E clone. Cell viability was shown as the percentage of the absorbance at $570 \mathrm{~nm}$ of D2-PrM/E clone relative to that of the parental 293 cells.

neither decrease in the percentage and intensity of positive cells detected by IFA nor decrease in the amounts of $\mathrm{E}$ protein in pellet lysates detected by Western blot analysis, suggesting that stable production of RSPs was successfully established in the $\mathrm{D} 2-\operatorname{PrM} / \mathrm{E}$ clone. In agreement with a previous report of stable clones continuously secreting dengue RSPs, the D2-PrM/E clone had the cell morphology and growth rate $(100 \%$ confluence 2 days after passage at a ratio of 1:6) similar to those of untransfected 293 cells, suggesting no obvious cytopathicity [37]. This was further supported by the observation of no decrease in cell viability of the established $\mathrm{D} 2-\operatorname{PrM} / \mathrm{E}$ clone assessed by an MTT cytotoxicity assay (Figure 1D).

\section{Construction of DENV2 subgenomic replicons and generation of infectious VLPS}

We next constructed a DENV2 subgenomic replicon by deleting the entire PrM and $\mathrm{E}$ gene from a previously reported DENV2 (Tonga/74 strain) infectious cNDA clone, p2 [28]. The resulting replicon, p2C102, contained genes encoding the Nterminal 102 residues of $\mathrm{C}$ protein (with the authentic cleavage site recognized by viral protease), the C-terminal 26 residues of $\mathrm{E}$ protein (encoding the signal sequence of NS1), and all the non-structural proteins (Figure 2). To examine whether p2C102 RNA can replicate in the D2-PrM/E clone, p2C102 RNA was electroporated to the D2-PrM/E clone, and RNA synthesis 


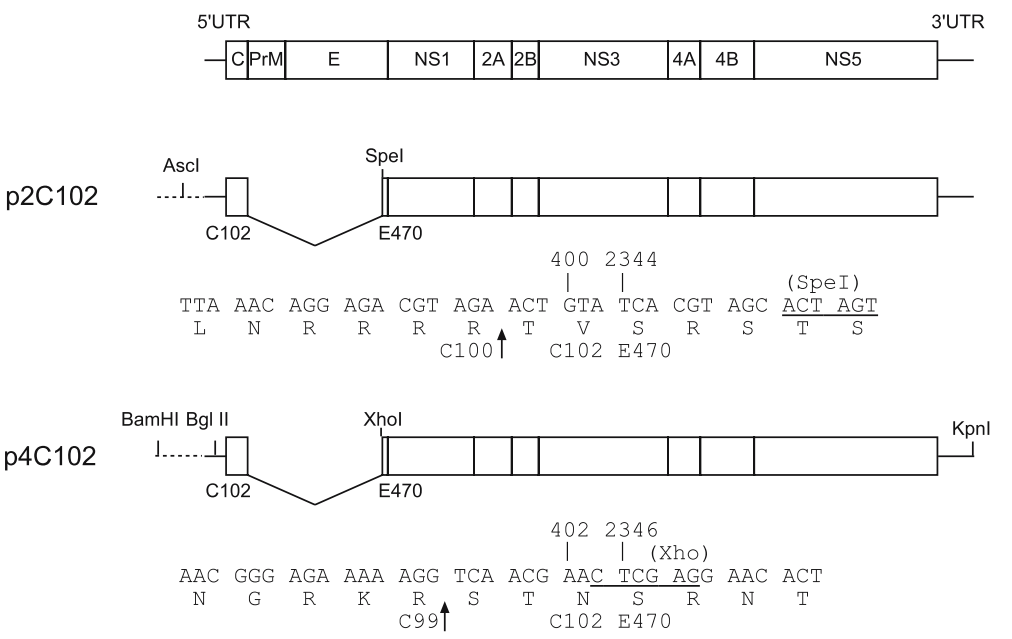

Figure 2. Schematic diagram of the dengue virus genome and subgenomic replicon constructs $\mathrm{p} 2 \mathrm{C} 102$ and $\mathrm{p} 4 \mathrm{C} 102$. Above each construct, the restriction enzyme sites used in the construction are shown. Below each construct, the sequences and numbers of both nucleotide and amino acid at the junction between $\mathrm{C}$ and $\mathrm{E}$ genes are shown $[28,29]$, with the restriction enzymes underlined and viral protease cleavage site of $\mathrm{C}$ protein indicated by arrow.

and protein expression were analyzed. As shown in Figure $3 \mathrm{~A}$, a band with the predicted size of $110-$ bp was seen in the p2C102 RNA-transfected but not in the mock-transfected cells or in the no RT control experiment, indicating that negative-sense RNA was synthesized by $\mathrm{p} 2 \mathrm{C} 102$. To examine the protein expression, cell lysates derived from p2C102 RNA-transfected cells were subjected to Western blot analysis using a anti-NS1 MAb, DB29-1. As shown in Figure 3B, a band of $38 \mathrm{KDa}$, the predicted size of NS1, was detected in the p2C102 RNA-transfected but not in the mock-transfected cells, indicating that NS1 was expressed by $\mathrm{p} 2 \mathrm{C} 102$. This was further supported by IFA using DB29-1 (Figure 3C). Taken together, these findings demonstrated that $\mathrm{p} 2 \mathrm{C} 102$ can replicate in the target cells.

To investigate whether p2C102 RNA can be packaged into infectious VLPs, pellets derived from culture supernatants of p2C102 RNA-transfected D2-PrM/E clone, which presumably contained VLPs, were inoculated to BHK-21 cells. After 2 days, positive cells were detected by IFA, indicating that infectious VLPs were generated by this packaging cell line, D2-PrM/E clone (Figure 3D). Inoculation of culture supernatants derived from the VLPs-infected BHK-21 cells to fresh BHK-21 cells did not result in any positive cell, consistent with the characteristics of oneround replication of VLPs (data not shown).

\section{Construction of DENV4 subgenomic replicons and generation of chimeric infectious VLPS}

Using a similar strategy, we generated a DENV4 subgenomic replicon, $\mathrm{p} 4 \mathrm{C} 102$, from a previously described DENV4 (814,669 strain) infectious clone, p2AXho (Figure 2) [30]. To examine whether p4C102 RNA can replicate in the D2-PrM/E clone, p4C102 RNA was electroporated to the D2-PrM/E clone, and RNA synthesis and protein expression were analyzed. As shown in Figure 4A, a band with the predicted size of 200-bp was seen in the p4C102 RNA-transfected as well as in the full-length infectious clone p4 RNA-transfected cells [29, 38], but not in the mock-transfected cells or in the no RT control experiment, indicating that negative-sense RNA was synthesized by p4C102 RNA-transfected cells. Western blot analysis revealed readily detectable NS1 band in the p4C102 RNA-transfected cells, though not as strong as the p4 RNA-transfected cells in this experiment, indicating that NS1 was expressed (Figure 4B). This was further supported by IFA (Figure 4C). Together, these findings demonstrated that $\mathrm{p} 4 \mathrm{C} 102 \mathrm{RNA}$ can replicate in the target cells. To investigate whether p4C102 RNA can also be packaged by the D2-PrM/E clone and produce chimeric infectious VLPs, pellets derived from the culture supernatants were inoculated to BHK-21 cells. After 2 days, positive cells were revealed by IFA using anti-NS1 Mab, indicating 


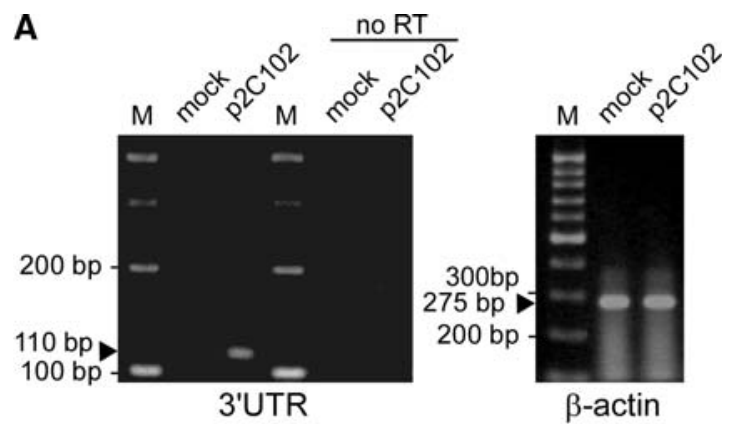

B

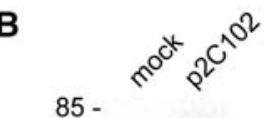

85



36 -

26 -

$20-$
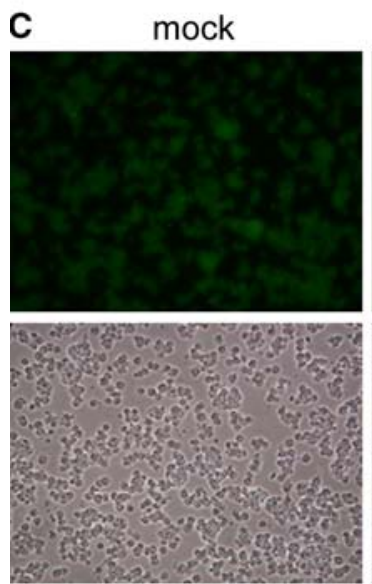

D
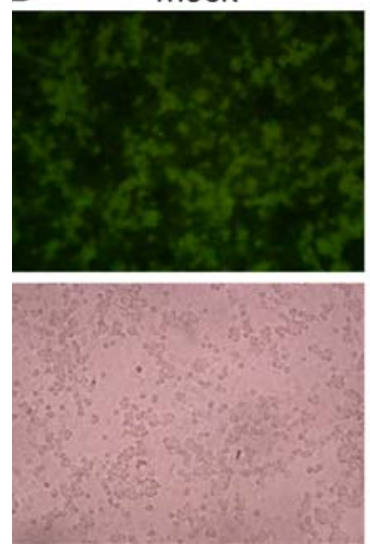
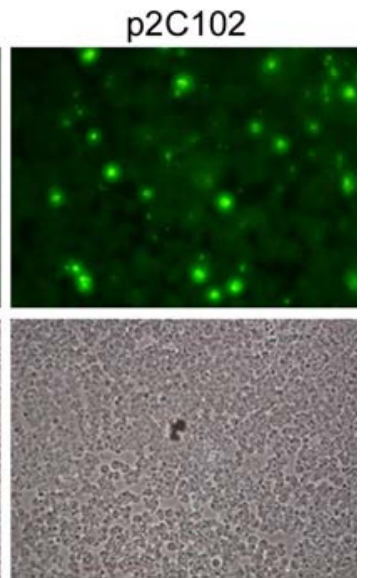

p2C102
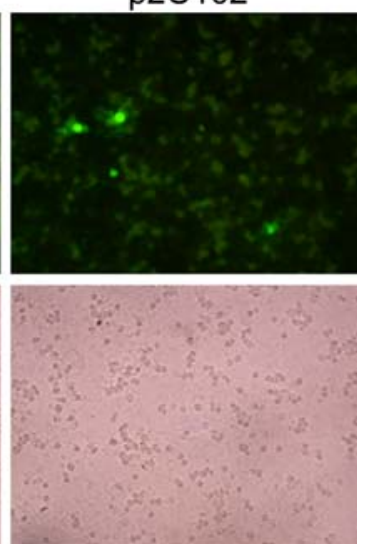

Figure 3. Replication of the DENV2 replicon p2C102 and generation of infectious VLPs. (A) RNA synthesis of p2C102. Total RNA derived from mock or p2C102 RNA-transfected $\mathrm{D} 2-\operatorname{PrM} / \mathrm{E}$ clone was subjected to RT using sense primer specific to 3'UTR or no RT, followed by PCR and agarose gel electrophoresis. RT-PCR using $\beta$-actin specific primers served as control. Arrow head indicates the size of predicted product, and M molecular size marker. (B) Protein expression of p2C102. Cell lysates derived from mock or p2C102 RNAtransfected D2-PrM/E clone were subjected to Western blot analysis by using anti-NS1 MAb, DB29-1. Arrow head indicates the NS1 protein. The size of molecular weight marker is shown in $\mathrm{kDa}$. (C) Protein expression in mock or p2C102 RNA-transfected D2-PrM/E clone was examined by IFA using DB29-1. (D) Generation of infectious VLPs. Pellets derived from culture supernatants of mock or p2C102 RNAtransfected D2-PrM/E clone were inoculated into BHK-21 cells, which were examined 48 h later by IFA using DB29-1. One representative experiment of three is shown.

that infectious chimeric VLPs were generated by D2-PrM/E clone (Figure 4D).

To demonstrate that the DENV4 replicon was indeed incorporated into the VLPs, pellets derived from culture supernatants of p4C102 RNA-transfected D2-PrM/E were subjected to RNA extraction, RT and PCR detecting viral genomic RNA in two regions, the $3^{\prime} \mathrm{UTR}$ and $\mathrm{C}$ gene. As shown in Figure 5A, a band with the predicted size of 200-bp in the $3^{\prime}$ UTR and a band with the predicted size of 170-bp in $\mathrm{C}$ gene were seen in the pellets derived from the p4C102 RNA-transfected and p4 RNAtransfected cells, but not in those from mocktransfected cells, indicating that the VLPs contained p4C102 replicon RNA. Moreover, Western blot analysis of pellet lysates using sera from a dengue patient [30] and from a rabbit immunized against DENV4 C protein revealed readily detectable PrM, $\mathrm{E}$ and $\mathrm{C}$ proteins in the pellets of $\mathrm{p} 4 \mathrm{C} 102 \mathrm{RNA}-$ and p4 RNA-transfected cells, indicating that DENV4 $\mathrm{C}$ protein was incorporated into the VLPs (Figure 5B). Electron microscopic examination of pellets derived from p4C102 RNA-transfected cells revealed particles with a diameter of $40-50 \mathrm{~nm}$, which was within the range of a flaviviral particle (40-60 nm) (Figure 5C) [4]. Taken together, these findings indicate successful incorporation of DENV4 replicon into the chimeric VLPs.

\section{Discussion}

With the application of recombinant DNA technology and reverse genetics, VLPs of several 

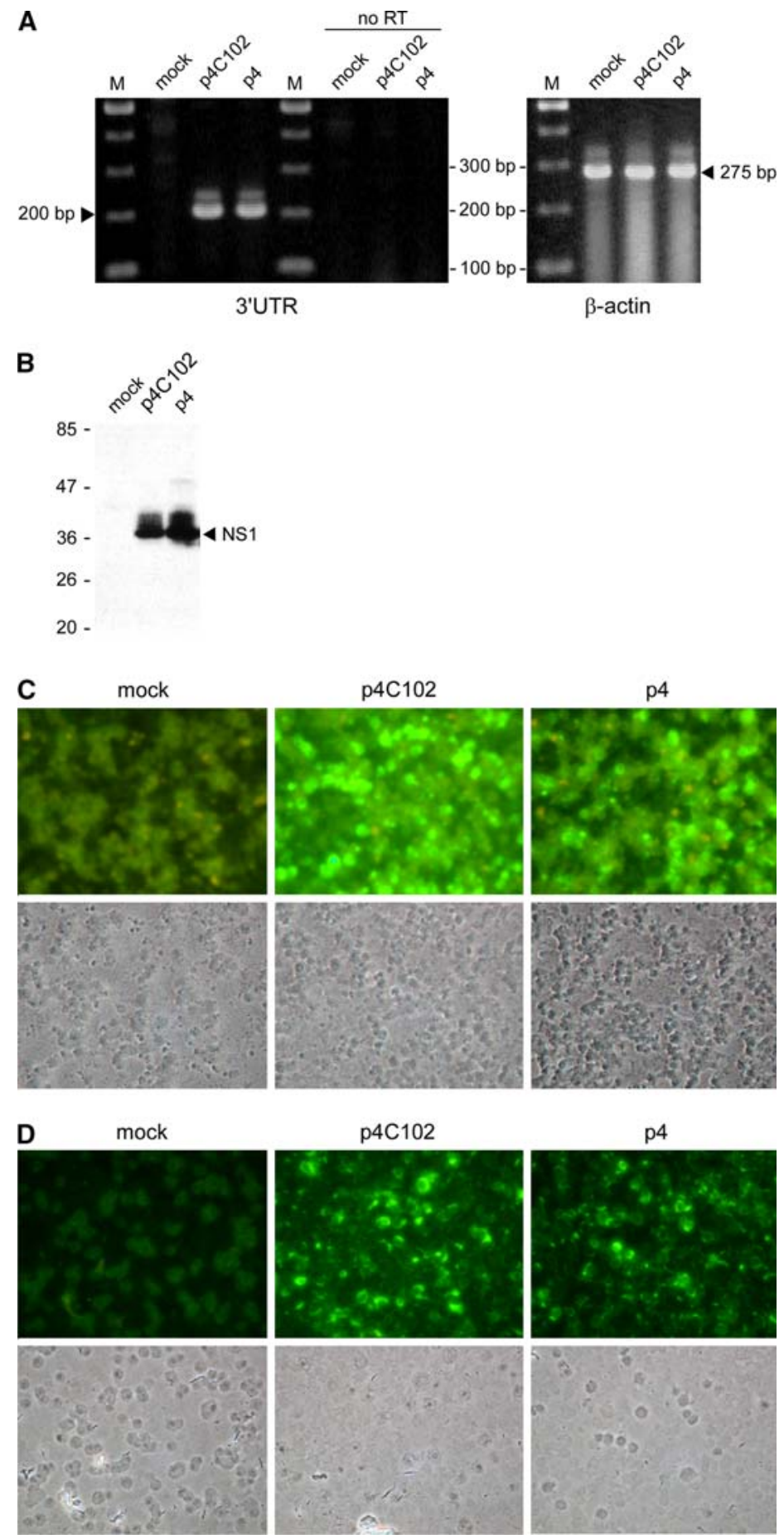

Figure 4. Replication of the DENV4 replicon p4C102 and generation of infectious VLPs. (A) RNA synthesis of p4C102. (B) Protein expression of p4C102. (C) Protein expression examined by IFA. (D) Generation of infectious VLPs. Total RNA, cell lysates, cells and pellets derived from mock, p4C102 RNA, or p4 RNA-transfected D2-PrM/E clone were subjected to analysis as described for Fig. 3. One representative experiment of three is shown. 
A


anti-DV
C
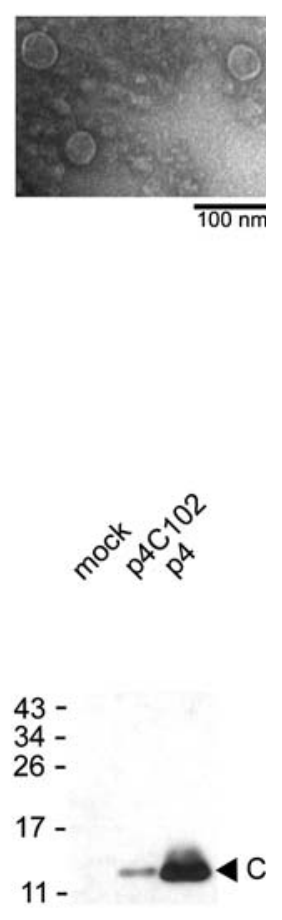

anti-C

Figure 5. Incorporation of DENV4 replicon into the chimeric VLPs. (A) Detection of DENV4 RNA in VLPs. RNA was extracted from pellets derived from mock, p4C102 RNA, or p4 RNA-transfected D2-PrM/E clone and subjected to RT using antisense primer specific to $3^{\prime} \mathrm{UTR}$ and $\mathrm{C}$ gene, followed by PCR and agarose gel electrophoresis. Arrow head indicates the size of predicted product, and $\mathrm{M}$ molecular size marker. (B) Proteins in the chimeric VLPs. Pellets derived from culture supernatants of mock, p4C102 RNA, or p4 RNA-transfected D2-PrM/E clone were subjected to Western blot analysis by using sera from a dengue patient (anti-DV) [30] and from a rabbit immunized against DENV4 C protein (anti-C). (C) Electron micrographs of the chimeric VLPs. An aliquot of pelleted particles were stained by uranyl acetate and examined under electron microscope. 100,000-fold magnification. Bar indicates $100 \mathrm{~nm}$.

flaviviruses thus established have been shown to be valuable for studying viral replication and inhibitors and promising for developing vectors of gene therapy and vaccines $[14,17,21-27,39-42]$. In this study, we have established a cell line stably producing RSPs of DENV2, which can successfully incorporate DENV2 replicon and produce single-round infectious VLPs. In addition to homologous incorporation, this cell line can also incorporate heterologous DENV4 replicon and produce infectious chimeric VLPs. To our knowledge,

this study reported for the first time VLPs containing replicons of dengue virus, which causes the most important and prevalent arboviral diseases worldwide.

Although chimeric dengue viruses have been generated from transfection of chimeric infectious cDNA clones, which contained $\mathrm{C} / \mathrm{PrM} / \mathrm{E}$ or $\operatorname{PrM} /$ $\mathrm{E}$ genes of one serotype and non-structural genes of another serotype in cis [30,43-45], the production and analysis of particles requires multiple rounds of successful amplification in the host cells, including RNA replication, encapsidation, assembly and release. Any defect in encapsidation that also inhibits particle formation and release would make the analysis of particles impossible. In contrast, our dengue $\operatorname{PrM} / \mathrm{E}$ expressing cell line, which produces particles continuously and packages replicon in trans, is particularly useful for studying the elements required for encapsidation. To demonstrate the potential application of this system to study encapsidation of DENV, we have generated another DENV4 replicon, p4C30 (Figure 6A), which contained only the N-terminal 30 amino acid residues of $\mathrm{C}$ protein corresponding to the cyclization sequences required for flavivirus replication, and examined its capability of generating infectious VLPs. After electroporation of p4C30 RNA into the D2-PrM/E clone, a band with the predicted size of 200-bp by RT-PCR and a band corresponding to NS1 by Western blot analysis were detected in the p4C30 RNA-transfected cells, indicating that p4C30 RNA can replicate in the D2-PrM/E clone (Figure 6B and data not shown). Inoculation of pellets derived from the p4C102 RNA-transfected cells and those from the p4C30 RNA-transfected cells into BHK21 cells revealed positive cells in the former but none in the latter, suggesting that amino acid residues from 31 to 101 of $\mathrm{C}$ protein were required for generation of infectious VLPs (Figure 6C). Further experiments are needed to delineate the minimal domain of $\mathrm{C}$ protein required for encapsidation.

Compared with the methods commonly used to generate VLPs, which involved two rounds of RNA transfection, namely, the first one introducing replicon RNA into cells transiently or in a stable clone and the second one introducing alphavirus replicon RNA to provide structural proteins $[9,17,22,24,25]$, our strategy of transfecting replicon to a packaging cell line 




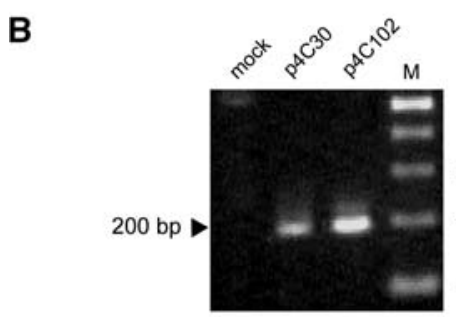

3'UTR


Figure 6. Incapable of generating infectious VLPs by DENV4 replicon p4C30. (A) Schematic diagram of the DENV4 replicon construct p4C30. The designation of the construct was as described in Fig. 2. (B) RNA synthesis of p4C30. (C) Infectivity of VLPs. Total RNA and pellets derived from mock, p4C30 RNA, or p4C102 RNA-transfected D2-PrM/E clone were subjected to analysis as described for Fig. 3. One representative experiment of three is shown.

expressing structural proteins avoids the problem of cytopathicity associated with some alphavirus replicons $[14,21]$. The lack of cytopathicity in our system was supported by the observations of no obvious change in cell morphology and growth rate. Moreover, an MTT cytotoxicity assay revealed no decrease in cell viability of the D2PrM/E clone, when compared with parental 293 cells which was not transfected with pCDJssD2J396 (Figure 1D). A closer examination of the expression of replicon and the infectivity of VLPs containing replicon revealed that the infectivity of VLPs correlated with the expression of the replicon, which was most likely related to the efficiency of RNA transfection (Figures 3, 4). Since the transfection efficiency of p2C102 RNA was lower than that of p4C102 RNA, the infectivity of VLPs derived from $\mathrm{p} 2 \mathrm{C} 102$ was lower than that derived from $\mathrm{p} 4 \mathrm{C} 102$. The highest titer of dengue VLPs derived from $\mathrm{p} 4 \mathrm{C} 102$ in this study, estimated by a previously described formula $[17,27]$, was $1.5 \times 10^{5} \mathrm{IU} / \mathrm{ml}$, which was lower than those reported for other flaviviruses $[14,17$, 21, 22, 24-26]. Further improvement in the efficiency of RNA transfection is needed to increase the titers of our VLPs.

During the past 25 years, there is a global resurgence of epidemic dengue with an increase in the frequency and number of cases with severe disease [1,2]. Without effective control measures for mosquitoes and available antiviral drugs, vaccination offers the best opportunity to lower 
the disease burden of dengue [46-48]. Currently, several tetravalent live attenuated dengue vaccines are being developed with the goal of attaining a balanced immunity to all four dengue serotypes [46-48]. Although improved immunogenicity and protective immunity in non-human primates have been recently reported for empirically and genetically engineered live attenuated tetravalent vaccines, respectively, reactogenicity such as fever or rash in more than $75 \%$ of recipients suggested that further attenuation is needed [48-51]. In this regard, VLPs of dengue virus containing replicon, which can complete single round of replication without obvious cytopathicity, in the tetravalent format could be an attractive alternative. Moreover, it has been shown that immunization with NS1 protein and passive transfer of anti-NS1 antibody can protect animals against flavivirus infection $[52,53]$. Our success in generating chimeric VLPs, which contain PrM and E proteins of one serotype and non-structural proteins of another serotype, suggesting the feasibility of adding the desired nonstructural proteins in the construction of VLPs. The capability of generating different chimeric dengue VLPs in the tetravalent format would further the potential of developing VLPs as dengue vaccine in the future.

\section{Acknowledgments}

We thank Dr. G. J. J. Chang at the Center for Disease Control and Prevention, Fort Collins for kindly providing the plasmid, pCBD2-2J-2-9-1, Dr. C. J. Lai at the National Institute of Health for the plasmid p2AXhoI, Dr. S. S. Whitehead at the National Institute of Health for the plasmids p2 and p4, Dr. H. C. Wu at the Institute of Cellular and Organismic Biology, Academia Sinica, Taipei, Taiwan for the Mab DB29-1. This work was supported by the National Science Council Taiwan (NSC95-2320-B-002-084-MY3).

\section{References}

1. Gubler D.J., Epidemic dengue/dengue hemorrhagic fever as a public health, social and economic problem in the $21 \mathrm{st}$ century. Trends Microbiol. 10: 100-103, 2002.

2. Guzman M.G. and Kouri G., Dengue: an update. Lancet Infect. Dis. 2: 33-42, 2002.
3. World Health Organization, Dengue Hemorrhagic Fever, Diagnosis, Treatment and Control, 2nd ed.,. World Health Organization, Geneva, 1997.

4. Lindenbach B.D. and Rice C.M., Flaviviridae: the viruses and their replication, In: Knipe D.M., Howley P.M. and Griffin D.E. (Eds.), Fields Virology. Lippincott William \& Wilkins, Philadelphia,PA, 2001, pp. 991-1041.

5. Ferlenghi I., Clarke M., Ruttan T., Allison S.L., Schalich J., Heinz F.X., Harrison S.C., Rey F.A. and Fuller S.D., Molecular organization of a recombinant subviral particle from tick-borne encephalitis virus. Mol. Cell 7: 593-602, 2001.

6. Konishi E., Pincus S., Paoletti E., Shope R.E., Burrage T. and Mason P.W., Mice immunized with a subviral particle containing the Japanese encephalitis virus $\mathrm{prM} / \mathrm{M}$ and $\mathrm{E}$ proteins are protected from lethal JEV infection. Virology 188: 714-720, 1992.

7. Schalich J., Allison S.L., Stiasny K., Mandl C.W., Kunz C. and Heinz F.X., Recombinant subviral particles from tickborne encephalitis virus are fusogenic and provide a model system for studying flavivirus envelope glycoprotein functions. J. Virol. 70: 4549-4557, 1996.

8. Blight K.J., Kolykhalov A.A. and Rice C.M., Efficient initiation of HCV RNA replication in cell culture. Science 290: 1972-1974, 2000.

9. Khromykh A.A., Replicon-based vectors of positive strand RNA viruses. Curr. Opin. Mol. Ther. 2: 555-569, 2000.

10. Khromykh A.A., Sedlak P.L. and Westaway E.G., Cis- and trans-acting elements in flavivirus RNA replication. J. Virol. 74: 3253-3263, 2000.

11. Lohmann V., Korner F., Koch J., Herian U., Theilmann L. and Bartenschlager R., Replication of subgenomic hepatitis C virus RNAs in a hepatoma cell line. Science 285: 110113, 1999.

12. Hewson R., RNA viruses: emerging vectors for vaccination and gene therapy. Mol. Med. Today 6: 28-35, 2000.

13. Schlesinger S., Alphavirus expression vectors. Adv. Virus Res. 55: 565-577, 2000.

14. Gehrke R., Ecker M., Aberle S.W., Allison S.L., Heinz F.X. and Mandl C.W., Incorporation of tick-borne encephalitis virus replicons into virus-like particles by a packaging cell line. J. Virol. 77: 8924-8933, 2003.

15. Hayasaka D., Yoshii K., Ueki T., Iwasaki T. and Takashima I., Sub-genomic replicons of Tick-borne encephalitis virus. Arch. Virol. 149: 1245-1256, 2004.

16. Holden K.L., Stein D.A., Pierson T.C., Ahmed A.A., Clyde K., Iversen P.L. and Harris E., Inhibition of dengue virus translation and RNA synthesis by a morpholino oligomer targeted to the top of the terminal $3^{\prime}$ stem-loop structure. Virology 344: 439-452, 2006.

17. Jones C.T., Patkar C.G. and Kuhn R.J., Construction and applications of yellow fever virus replicons. Virology 331: 247-259, 2005.

18. Khromykh A.A. and Westaway E.G., Subgenomic replicons of the flavivirus Kunjin: construction and applications. J. Virol. 71: 1497-1505, 1997.

19. Pang X., Zhang M. and Dayton A.I., Development of dengue virus type 2 replicons capable of prolonged expression in host cells. BMC Microbiol. 1: 18, 2001.

20. Shi P.Y., Tilgner M. and Lo M.K., Construction and characterization of subgenomic replicons of New York strain of West Nile virus. Virology 296: 219-233, 2002.

21. Harvey T.J., Liu W.J., Wang X.J., Linedale R., Jacobs M., Davidson A., Le T.T., Anraku I., Suhrbier A., Shi P.Y. and 
Khromykh A.A., Tetracycline-inducible packaging cell line for production of flavivirus replicon particles. J. Virol. 78: 531-538, 2004.

22. Khromykh A.A., Varnavski A.N. and Westaway E.G., Encapsidation of the flavivirus kunjin replicon RNA by using a complementation system providing Kunjin virus structural proteins in trans. J. Virol. 72: 5967-5977, 1998.

23. Pierson T.C., Sanchez M.D., Puffer B.A., Ahmed A.A., Geiss B.J., Valentine L.E., Altamura L.A., Diamond M.S. and Doms R.W., A rapid and quantitative assay for measuring antibody-mediated neutralization of West Nile virus infection. Virology 346: 53-65, 2006.

24. Puig-Basagoiti F., Deas T.S., Ren P., Tilgner M., Ferguson D.M. and Shi P.Y., High-throughput assays using a luciferase-expressing replicon, virus-like particles, and full-length virus for West Nile virus drug discovery. Antimicrob. Agents Chemother. 49: 4980-4988, 2005.

25. Scholle F., Girard Y.A., Zhao Q., Higgs S. and Mason P.W., Trans-packaged West Nile virus-like particles: infectious properties in vitro and in infected mosquito vectors. J. Virol. 78: 11605-11614, 2004.

26. Varnavski A.N. and Khromykh A.A., Noncytopathic flavivirus replicon RNA-based system for expression and delivery of heterologous genes. Virology 255: 366-375, 1999.

27. Yoshii K., Hayasaka D., Goto A., Kawakami K., Kariwa H. and Takashima I., Packaging the replicon RNA of the Far-Eastern subtype of tick-borne encephalitis virus into single-round infectious particles: development of a heterologous gene delivery system. Vaccine 23: 3946-3956, 2005.

28. Blaney J.E. Jr., Hanson C.T., Hanley K.A., Murphy B.R. and Whitehead S.S., Vaccine candidates derived from a novel infectious cDNA clone of an American genotype dengue virus type 2. BMC Infect. Dis. 4: 39, 2004.

29. Lai C.J., Zhao B.T., Hori H. and Bray M., Infectious RNA transcribed from stably cloned full-length cDNA of dengue type 4 virus. Proc. Natl. Acad. Sci. USA 88: 5139-5143, 1991.

30. Bray M. and Lai C.J., Construction of intertypic chimeric dengue viruses by substitution of structural protein genes. Proc. Natl. Acad. Sci. USA 88: 10342-10346, 1991.

31. Wang W.K., Chen H.L., Yang C.F., Hsieh S.C., Juan C.C., Chang S.M., Yu C.C., Lin L.H., Huang J.H. and King C.C., Slower rates of clearance of viral load and viruscontaining immune complexes in patients with dengue hemorrhagic fever. Clin. Infect. Dis. 43: 1023-1030, 2006.

32. Henchal E.A., Gentry M.K., McCown J.M. and Brandt W.E., Dengue virus-specific and flavivirus group determinants identified with monoclonal antibodies by indirect immunofluorescence. Am. J. Trop. Med. Hyg. 31: 830-836, 1982.

33. Chang G.J., Hunt A.R., Holmes D.A., Springfield T., Chiueh T.S., Roehrig J.T. and Gubler D.J., Enhancing biosynthesis and secretion of premembrane and envelope proteins by the chimeric plasmid of dengue virus type 2 and Japanese encephalitis virus. Virology 306: 170-180, 2003.

34. Chang G.J., Hunt A.R. and Davis B., A single intramuscular injection of recombinant plasmid DNA induces protective immunity and prevents Japanese encephalitis in mice. J. Virol. 74: 4244-4252, 2000.

35. Chen C. and Okayama H., High-efficiency transformation of mammalian cells by plasmid DNA. Mol. Cell Biol. 7: 2745-2752, 1987.

36. Wang W.K., Lee C.N., Kao C.L., Lin Y.L. and King C.C., Quantitative competitive reverse transcription-PCR for quantification of dengue virus RNA. J. Clin. Microbiol. 38: 3306-3310, 2000.

37. Holmes D.A., Purdy D.E., Chao D.Y., Noga A.J. and Chang G.J., Comparative analysis of immunoglobulin M (IgM) capture enzyme-linked immunosorbent assay using virus-like particles or virus-infected mouse brain antigens to detect IgM antibody in sera from patients with evident flaviviral infections. J. Clin. Microbiol. 43: 3227-3236, 2005.

38. Durbin A.P., Karron R.A., Sun W., Vaughn D.W., Reynolds M.J., Perreault J.R., Thumar B., Men R., Lai C.J., Elkins W.R., Chanock R.W., Murphy B.R. and Whitehead S.S., Attenuation and immunogenicity in humans of a live dengue virus type- 4 vaccine candidate with a 30 nucleotide deletion in its $3^{\prime}$-untranslated region. Am. J. Trop. Med. Hyg. 65: 405-413, 2001.

39. Anraku I., Harvey T.J., Linedale R., Gardner J., Harrich D., Suhrbier A. and Khromykh A.A., Kunjin virus replicon vaccine vectors induce protective CD $8+$ T-cell immunity. J. Virol. 76: 3791-3799, 2002.

40. Harvey T.J., Anraku I., Linedale R., Harrich D., Mackenzie J., Suhrbier A. and Khromykh A.A., Kunjin virus replicon vectors for human immunodeficiency virus vaccine development. J. Virol. 77: 7796-7803, 2003.

41. Lo M.K., Tilgner M. and Shi P.Y., Potential highthroughput assay for screening inhibitors of West Nile virus replication. J. Virol. 77: 12901-12906, 2003.

42. Rossi S.L., Zhao Q., O’Donnell V.K. and Mason P.W., Adaptation of West Nile virus replicons to cells in culture and use of replicon-bearing cells to probe antiviral action. Virology 331: 457-470, 2005.

43. Chen W., Kawano H., Men R., Clark D. and Lai C.J., Construction of intertypic chimeric dengue viruses exhibiting type 3 antigenicity and neurovirulence for mice. J.Virol. 69: 5186-5190, 1995.

44. Huang C.Y., Butrapet S., Pierro D.J., Hang G.J.J., Hunt A.R., Bhamarapravati N., Gubler D.J. and Kinney R.M., Chimeric dengue type 2 (vaccine strain PDK-53)/dengue type 1 virus as a potential candidate dengue type 1 virus vaccine. J. Virol. 74: 3020-3028, 2000.

45. Huang C.Y., Butrapet S., Tsuchiya K.R., Bhamarapravati N., Gubler D.J. and Kinney R.M., Dengue 2 PDK-53 virus as a chimeric carrier for tetravalent dengue vaccine development. J. Virol. 77: 11436-11447, 2003.

46. Blaney J.E. Jr., Durbin A.P., Murphy B.R. and Whitehead S.S., Development of a live attenuated dengue virus vaccine using reverse genetics. Viral Immunol. 19: 10-32, 2006.

47. Lai C.J. and Monath T.P., Chimeric flaviviruses: novel vaccines against dengue fever, tick-borne encephalitis, and Japanese encephalitis. Adv. Virus Res. 61: 469-509, 2003.

48. Pugachev K.V., Guirakhoo F. and Monath T.P., New developments in flavivirus vaccines with special attention to yellow fever. Curr. Opin. Infect. Dis. 18: 387-394, 2005.

49. Sabchareon A., Lang J., Chanthavanich P., Yoksan S., Forrat R., Attanath P., Sirivichayakul C., Pengsaa K., Pojjaroen-Anant C., Chambonneau L., Saluzzo J.F. and Bhamarapravati N., Safety and immunogenicity of a three dose regimen of two tetravalent live-attenuated dengue vaccines in five- to twelve-year-old Thai children. Pediatr. Infect. Dis. J. 23: 99-109, 2004.

50. Blaney J.E. Jr., Matro J.M., Murphy B.R. and Whitehead S.S., Recombinant, live-attenuated tetravalent dengue virus vaccine formulations induce a balanced, broad, and protective neutralizing antibody response against each of 
the four serotypes in rhesus monkeys. J. Virol. 79: 55165528, 2005.

51. Durbin A.P., Whitehead S.S., McArthur J., Perreault J.R., Blaney J.E. Jr., Thumar B., Murphy B.R. and Karron R.A., rDEN4delta30, a live attenuated dengue virus type 4 vaccine candidate, is safe, immunogenic, and highly infectious in healthy adult volunteers. J. Infect. Dis. 191: 710-718, 2005.

52. Falgout B., Bray M., Schlesinger J.J. and Lai C.J., Immunization of mice with recombinant vaccinia virus expressing authentic dengue virus nonstructural protein NS1 protects against lethal dengue virus encephalitis. J. Virol. 64: 4356-4363, 1990.

53. Schlesinger J.J., Brandriss M.W., Cropp C.B. and Monath T.P., Protection against yellow fever in monkeys by immunization with yellow fever virus nonstructural protein NS1. J. Virol. 60: 1153-1155, 1986. 